\title{
Problem of the direct quantum-information transformation of chemical substance
}

\author{
Vasil Penchev* \\ *Bulgarian Academy of Sciences: Institute of Philosophy and Sociology: Dept. of Logical Systems and Models; vasildinev@gmail.com
}

\begin{abstract}
Arthur Clark and Michael Kube-McDowell ("The Triger", 2000) suggested the sci-fi idea about the direct transformation from a chemical substance to another by the action of a newly physical, "Trigger" field. Karl Brohier, a Nobel Prize winner, who is a dramatic persona in the novel, elaborates a new theory, re-reading and re-writing Pauling's "The Nature of the Chemical Bond"; according to Brohier: "Information organizes and differentiates energy. It regularizes and stabilizes matter. Information propagates through matter-energy and mediates the interactions of matter-energy." Dr Horton, his collaborator in the novel replies: "If the universe consists of energy and information, then the Trigger somehow alters the information envelope of certain substances -“.

"Alters it, scrambles it, overwhelms it, destabilizes it" Brohier adds.

There is a scientific debate whether or how far chemistry is fundamentally reducible to quantum mechanics. Nevertheless, the fact that many essential chemical properties and reactions are at least partly representable in terms of quantum mechanics is doubtless. For the quantum mechanics itself has been reformulated as a theory of a special kind of information, quantum information, chemistry might be in turn interpreted in the same terms.
\end{abstract}

\section{INTRODUCTION}

Chemistry has seemed to be underlain by quantum mechanics since the age of the "old" quantum mechanics suggested by Niels Bohr and others to explain the complex build of atom. The periodic table of all chemical elements invented by D. Mendeleev could be explained elementary by the unique electron configuration ("shell") featuring any chemical element. The chemical bonds are unambiguously determined by the corresponding electron configurations [1], [2], [3].

The boundary between chemistry and physics was overcome modifying the atoms of a chemical element into another only by physical action such as the bombardment of nuclei by high-energy particles. New chemical elements, which cannot be found in nature because of their short half-life, were synthesized artificially. The phenomena of radioactivity linked chemistry and quantum mechanics [3], [4], [5].

Independently of all those exceptional successes and the corresponding series of Nobel prizes, the further development of chemistry and quantum mechanics move away them from each other [3], [6].

Only one of the four known physical interactions, namely the electromagnetic one, refers to chemistry being both strong
Wave function, the fundamental concept of quantum mechanics, can be equivalently defined as a series of qubits, eventually infinite. A qubit, being defined as the normed superposition of the two orthogonal subspaces of the complex Hilbert space, can be interpreted as a generalization of the standard bit of information as to infinite sets or series. All "forces" in the Standard model, which are furthermore essential for chemical transformations, are groups $[U(1), S U(2), S U(3)]$ of the transformations of the complex Hilbert space and thus, of series of qubits.

One can suggest that any chemical substances and changes are fundamentally representable as quantum information and its transformations. If entanglement is interpreted as a physical field, though any group above seems to be unattachable to it, it might be identified as the "Triger field". It might cause a direct transformation of any chemical substance by from a remote distance. Is this possible in principle?

Key words: axiom of choice; axiom of induction; axiom of transfinite induction; eidetic, phenomenological and transcendental reduction; epoché; Gödel mathematics; Hilbert mathematics; information; quantum mechanics, quantum information; phenomenology; principle of universal mathematizability

enough and acting at any distance [7]. The strong interaction though "strong" cannot overcome the distance between the atoms or molecules. The gravitational one can really do this, but it is too weak to cause any meaningful effect. At last, the weak one combines both disadvantages.

The chemical bonds need only electron configurations to be explained paying no attention to all the rest elementary particles [7], [8].

The properties of chemical compounds especially organic ones depend essentially on the molecular structures [8], [9], and the thermodynamic properties of huge ensembles [10], on the impurities as well [11].

The chemical reactions depend on concentrations, thermodynamic quantities, catalysts, etc. [8], [12], [13], [14], [15], [16].

All those enumerated ingredients or conditions do not seem to refer directly to quantum mechanics though they are exceptionally essential for chemical cognition though many aspects of chemical reaction admit quantum explanation [17], [18], [19] including even catalysis [20]. 
However, quantum mechanics offers a universal viewpoint to all chemical elements or compounds being quantum systems as all in the material world [22], [21] They can be exhaustedly described by their wave functions, which are modelled as elements (or "points") in the separable complex Hilbert space. Then any physical quantity is described by a corresponding self-adjoint operator changing only the probabilities corresponding to one and the same values for the quantity at issue to be measured. All self-adjoint operators share the property of unitarity interpretable of energy conservation.

The chemical reactions might be defined by arbitrary operators on the separable complex Hilbert space, among which the self-adjoint ones are only a quite particular case. That viewpoint considers physics as a particular case of chemistry rather than the opposite. Quantum mechanics is what might justify that chemical "ideology" of the being.

All known until now physical interactions are suggested to be able to be described as quantum fields where a certain wave function is attached to any point of space-time. This is experimentally well-confirmed as to the strong, weak, and electromagnetic interaction unified in their joint Standard model, and it is yet a hypothesis as to gravity.

One can assume a special kind of generalized quantum field acting directly to the separable complex Hilbert space rather than indirectly by the meditation of space-time as in the known quantum fields in physics. It will be reducible to the above known quantum fields in space-time in the case of selfadjoint operators, but furthermore it would include the general case of arbitrary operators on the separable complex Hilbert space, i.e. all quantum representations of any chemical reactions. Its action might cause the direct change of any chemical substance into another at a distance without any mediation of any chemical reaction.

That theoretical option admitted by quantum mechanics is explored and described in the sci-fi novel "The Trigger" by Arthur Clark and Michael Kube-McDowell (2000). That kind of hypothetical quantum field is called there "Trigger field". It was found occasionally in the novel after it had caused bursts in all weapons and ammunition in a certain radius because of the change of their chemical contains.

Though being only a sci-fi idea, it seems to rest on serious research therefore being well-justifiable as follows. Furthermore, information underlies energy and matter according to the conception of the novel. The Trigger field changes directly the information base of any energetic or material entity such as any chemical substance therefore transforming it into another according to the applied quality and quantity of that field.

Consequently, the Trigger field is meant and expressively emphasized by the authors as a field of information directly changing the information featuring any chemical substance. Furthermore, physics is meant to be a particular case of chemistry in the conceptual framework of a new fundamental theory elaborated by one of the personages of the novel, Karl Brohier, a Nobel Prize winner.

What might correspond to the Trigger field in the framework of contemporary science is entanglement and the theory of quantum information studying the phenomena of entanglement.
Quantum information is a generalization of information introduced by quantum mechanics to reformulate its concepts, quantities and equations in terms of information. Quantum information is a quantity measured in qubits just as information is measured in bits.

A bit is defined as the choice between two equally probable alternatives, and a qubit can be equivalently defined as its generalization as the choice between an infinite set of alternatives.

Its original formulation in theory of quantum information means the normed superposition of two orthogonal ${ }^{1}$ subspaces of the separable complex Hilbert space. Thus the separable complex Hilbert space itself can be represented as a series of "empty qubits", in each of which can be "recorded" a value representing a normed pair of complex numbers. Then any wave function would be a certain value of the free variable of quantum information as what the separable complex Hilbert space can be considered. The state of any quantum system being always a wave function can be exhaustedly represented as a value of quantum information.

The phenomena of entanglement can be defined as the direct interaction of quantum fields, which are not independent of each other in at least one space-time point. If they are independent of each other, their corresponding Hilbert space are orthogonal subspaces of the common Hilbert space of their joint system, and the latter in turn is decomposable into a tensor product of the compound Hilbert spaces.

One can discuss what happens in a certain space-time point, in which two or more dependable quantum fields are available and thus interact with each other.

Most generally, the probability of that point to be randomly chosen after measurement will depend nonlinearly on all constituting quantum subfields. If the interacting quantum fields are only two, any pair corresponding qubits will generate a new resultative qubits referring to the joint resultative quantum filed, after which the values of the new qubit can be elementarily calculated as the sums of the values of the initial qubits and the phase difference (rotation) between them.

This means that the wave function in an arbitrary spacetime point can be arbitrarily changed because of the action of another or other quantum field(s).

Furthermore, any standalone quantum field, which is not generated by a certain quantum entity having energy and eventually mass at rest, is not yet known or found. Anyway, principles or causes not to exist that standalone quantum field without any energetic carrier are not known, too.

Then, in the framework of contemporary physical knowledge, the removing of the carriers of quantum fields generating entanglement a great distance away will restore the initial wave function, for example, that of a single chemical substance. In other words, the stable modification of a wave function such as that in a chemical compound in comparison with its components needs a corresponding stable space-time aggregation to be constituted to be able to guarantee the constant entanglement of the constituting wave function. Chemical bond based on two electrons in one and same state

\footnotetext{
${ }^{1}$ Any two disjunctive subspaces of the separable complex Hilbert space are orthogonal.
} 
(i.e. one and the same wave function) realizes that necessary aggregate, but it does not exhaust the properties of the compound, which can be explained only by the complete modification of the constituting wave functions into a single and entangled one.

The attempt for the properties of chemical compound to be explained thoroughly by the initial properties of the ingredient and the chemical bonds between them should fail. It should be displaced by the complex nonlinear interaction of the constituent wave functions into entanglement conditioning properly the new and quite different properties of the compound.

Furthermore, entanglement can well explain how the catalysts act. They modify by entanglement the wave function at least of one of the ingredients of a chemical reaction, however without to constitute (long-time) chemical bounds. The modified wave function is already much abler to be further modified and held by chemical bonds. The catalysts go out of the reaction unchanged.

Entanglement conserves energy-momentum rather than energy and momentum separately. This may explain the way, in which chemical energy interacts immediately with the thermodynamic quantities such as pressure, temperature, volume, mechanical energy and differently defined energies in the course of the reaction.

One can consider all quantum particles within a generalization of 'chemical compound' where the necessary space-time aggregation for stable entanglement is realized by any interaction and fundamental particles able to do that rather than only electromagnetic one and electrons as in the chemical compound in a narrow sense. Indeed, the strength and infinite range of electromagnetic interaction complemented by the atom structure including an external electron shell, which can be shared constituting chemical bonds, assists much for that variety of chemical compounds on the macroscopic scale studied by classical science and human experience.

Similarly, strong and weak interactions as well as electromagnetic one, out of chemical compounds in a narrow sense as above, are able also to generate more or less stable compounds held of corresponding bonds (which can be seen as generalized chemical bonds). Independently of the difference in the kind of bonds, they share the same essence to modify the properties for the new joint entangled wave function.

Thus one can introduce a "chemistry of weak or strong interaction", or electromagnetic interaction out of the standard theory of chemical compounds.

The paper is organized as follows. Section 2 introduces a few basic concepts for the reinterpretation of quantum mechanics in terms of quantum information. Section 3 introduces the concept of entanglement and the way, in which it allows for chemical compound and the meaning of chemical bond to be reinterpreted. Section 4 explains the action of catalysts and thermodynamic conditions of chemical reaction on the base of entanglement. Section 5 discusses the generalization of the concept of chemical compound onto the area of all elementary particles studied by physics. Section 6 consider the direct question whether the "Trigger field" described by A. Clark and M. Kube-McDowell might exist in nature or be created artificially. Section 7 is devoted to philosophical or metaphysical conclusions and methodological corollaries. The last Section 8 summarizes the paper from the viewpoint of future research.

\section{QUANTUM MECHANICS IN TERMS OF QUANTUM INFORMATION}

The set of all complex numbers, $\boldsymbol{C}$ is granted. Then the corresponding set of all subset of $\boldsymbol{C}$ is the separable complex Hilbert space $\mathcal{H}$.

There is one common and often met identification of $\mathcal{H}$ with the set $\mathbb{H}$ of all ordinals of $\mathcal{H}$, which rests on the identification of any set with its ordinal. However, if any ordinal is identified as a certain natural number, and all natural numbers in Peano arithmetic are finite ${ }^{2}, \mathcal{H}$ and $\mathbb{H}$ should not be equated, for $\mathcal{H}$ includes actually infinite subsets $^{3}$ of $2^{C}$. Here "actually infinite subset" means 'set infinite in the sense of set theory".

Furthermore, $\mathcal{H}$ is identified as the set $\mathbf{H}$ of all wellordered sets which elements are elements of some set of $2^{C}$, i.e. in other words, the elements of $2^{C}$ considered as classes of equivalency in ordering are differed in ordering within any class of that ordering.

Those distinctions can be illustrated by the two basic interpretations of $\mathcal{H}:(1)$ as the vectors of n-dimensional complex generalization of the usual 3D real Euclidean space, isomorphic to $\mathbf{H}$, and (2) as the squarely integrable functions, isomorphic to $\mathcal{H}$. The latter adds to the former unitarity (unitary invariance), which is usually interpreted as energy conservation in their application in quantum mechanics. Back seen, energy conservation is a physical equivalent of both (3) equivalence afrer ordering and (4) actual infinity, i.e. to (5) the concept of ordinal number in set theory.

On the contrary, once one does not involves energy conservation, e.g. generalizing it to energy-momentum conservation as in the theory of general relativity or that of entanglement, $\mathbf{H}$ rather than $\mathcal{H}$ is what should be used unlike quantum mechanics based on $\mathcal{H}$, and actual infinity avoided or at least precisely thought before utilizing.

Furthermore, (6) the relation between $\mathcal{H}$ and $\boldsymbol{H}$ can be interpreted as the 3D Euclidean space under (7) the additional condition of cyclicality (reversibility) of $\boldsymbol{H}$ conventionally identifying the first "infinite" element with the "first" element of any (trans)finite well-ordering. Indeed, the axiom of induction in Peano arithmetic does not admit infinite natural numbers ${ }^{4}$. If one needs to reconcile both finite and transfinite induction to each other, the above condition is sufficient.

It should be chosen for Poincaré's conjecture [@] proved by G. Perelman [@]. If that condition misses, the topological structure is equivalent to any of both almost disjunctive domains $^{5}$ of Minkowski's space of special relativity ${ }^{6}$ rather

\footnotetext{
2 This is a property implied by the axiom of induction.

${ }^{3}$ Here "actually infinite subset" means "set infinite in the sense of set theory".

${ }^{4} 1$ is finite. The successor of any finite natural number is finite. Consequently, all natural numbers are finite for the axiom of induction.

${ }^{5}$ They are almost disjunctive as share the light cone.

${ }^{6}$ Indeed, special relativity is a causal theory, which excludes the reverse causality implied by cyclicality.
} 
than to a 4D Euclidean ball. The two domains of Minkowski space $\mathcal{M}$ can be interpreted as two opposite, "causal directions" resulting in both reversibility of the 3D Euclidean space and topological structure of the above 4D ball.

The relation between $\mathcal{H}$ and $\boldsymbol{H}$ generates any of the two areas of $\mathcal{M}$ as follows. Both unitarity of $\mathcal{H}$ and non-unitarity of $\boldsymbol{H}$ for any ordinal $n$ and any well-ordering of length $n$ are isomorphic to a 3D Euclidean sphere ${ }^{7}$ with the radius $r(n)$. All those spheres represent the area at issue.

That construction can be interpreted physically as well. Energy (E) conservation as unitarity represents the class of equivalence of any ordinal $n$. If the concept of physical force (F) is introduced as any reordering, i.e. the relation between any two elements of the above class, it can be reconciled with energy conservation (unitarity) by the quantity of distance (x) in units of elementary permutations for the reordering so that $F \cdot x=E$.

Back seen, both (6) and (7) implies Poincaré's conjecture and thus offer another way of its proof.

One can discuss the case where $\mathcal{H}$ is identified with $\boldsymbol{H}$ and what it implies. Then (8) the axiom of induction in Peano arithmetic should be replaced by transfinite induction correspondingly to (4) above, and (9) the statistical ensemble of well-orderings (as after measurement in quantum mechanics) should be equated to the set of the same elements (as the coherent state before measurement in quantum mechanics) for (3) above.

In fact, that is the real case in quantum mechanics for unitarity as energy conservation is presupposed. Then (8) implies the theorems of absence of hidden variables in quantum mechanics [@, @], i.e. a kind of mathematical completeness interpretable as the completeness of quantum mechanics vs. Einstein, Podolsky, and Rosen's hypothesis of the incompleteness of quantum mechanics [@]:

The (8) and (9) together imply the axiom of choice. Indeed, the coherent state (the unordered set of elements) excludes any well-ordering for the impossibility of hidden variables implied by (8). However, it can be anyway well-ordered for (9). This forces the well-ordering principle ("theorem") to be involved, which in turn to the axiom of choice.

Furthermore, $\mathcal{H}$ can be represented as all sets of qubits.

A qubit is defined in quantum mechanics and information as the (10) normed superposition of two orthogonal ${ }^{8}$ subspaces of $\mathcal{H}$ :

$$
Q \stackrel{\text { def }}{=} \alpha|0\rangle+\beta|1\rangle
$$

$|0\rangle,|1\rangle$ are the two orthogonal subspaces of $\mathcal{H}$.

$\alpha, \beta \in C:|\alpha|^{2}+|\beta|^{2}=1$.

Then, (11) $\mathrm{Q}$ is isomorphic to a unit 3D Euclidean ball, in which two points in two orthogonal great circles ate chosen so that the one of them (the corresponding to the coefficient $\beta$ ) is on the surface of the ball.

That interpretation is obvious mathematically. It makes sense physically and philosophically for the above consideration of space as the relation of $\mathcal{H}$ and $\boldsymbol{H}$.

Now, it can be slightly reformulated and reinterpreted as the joint representability of $\mathcal{H}$ and $\boldsymbol{H}$, and thus their unifiablity in terms of quantum information.

\footnotetext{
${ }^{7}$ This means the surface of a 3D Euclidean ball.

${ }^{8}$ Any two disjunctive subspaces of $\mathcal{H}$ are orthogonal to each other.
}

Particularly, any theory of quantum information, including quantum mechanics as far as it is so representable, admits the coincidence of model and reality: right a fact implied by the impossibility of hidden variables in quantum mechanics for any hidden variable would mean a mismatch of model and reality.

$\boldsymbol{H}$ can be interpreted as an equivalent series of qubits for any two successive axes of $\boldsymbol{H}$ are two orthogonal subspaces of $\mathcal{H}$ :

$\left\{C_{j}\right\} \in \boldsymbol{H}$; then (12) any successive pair $\left(C_{j}, C_{j+1}\right)=Q_{j+1}$; $Q_{j+1} \in \boldsymbol{Q}$ under the following conditions:

$$
\text { (13) } \alpha_{j+1}=\frac{C_{j}}{\sqrt{\left(C_{j}\right)^{2}+\left(C_{j+1}\right)^{2}}} ; \beta_{j+1}=\frac{C_{j+1}}{\sqrt{\left(C_{j}\right)^{2}+\left(C_{j+1}\right)^{2}}} ;
$$$$
\text { (14) } \alpha_{1}=0 ; \beta_{1}=\frac{C_{1}}{\left|C_{1}\right|} \text {; }
$$

(15) If both $C_{j}, C_{j+1}=0, \alpha_{j+1}=0, \beta_{j+1}=1$.

(14) and (15) are conventional, chosen rather arbitrarily only to be conserved a one-to-one mapping between $\boldsymbol{H}$ and $\boldsymbol{Q}$.

$\boldsymbol{Q}$ is intendedly constructed to be ambivalent to unitarity for any qubit is internally unitary, but the series of those is not. Furthermore, one can define n-bit where a qubit is 2-bit therefore transforming unitarily any non-unitary n-series of complex nubers. The essence of that construction is the double conservation between the two pairs: "within - out of" and "unitarity - nonunitarity".

That conservation is physical and informational, in fact, and will be discussed in detail in the next paragraph. The simultaneous choice between many alternatives being unitary and thus physically interpretable is equated to a series of elementary or at least more elementary choices. Then, the visible as physical inside will look like the mental outside and vice versa. If a wholeness such as the universe is defined to contain internally its externality, this can be modeled anyway consistently equating the non-unitary "mental" and unitary "physical" representations in the framework of a relevant physical and informational conservation.

$\mathcal{H}$ can be furthermore interpreted as all possible pairs of characteristic functions of independent probability distributions and thus, of all changes of probability distributions of the state of a system, e.g. a quantum system.

Practically all probability distributions and their characteristic functions of the states of real systems are continuous and even smooth as usual. The neighboring values of probability implies the neighborhood of the states. Thus the smoothness of probability distribution implies a well-ordering and by the meditation of it, a kind of causality: the probability of the current state cannot change jump-like.

This is an expression of a deep mathematical dependence (or invariance) of the continuous (smooth) and discrete. The probability distribution can mediate between them as follows:

$\mathcal{H}$ can be defined as the sets of the ordinals of $\boldsymbol{H}$ where a representative among any subset of the permutations (wellorderings) of $n$ elements is chosen according a certain and thus constructive rule. That rule in the case in question is to be chosen that permutation (well-ordering), the probability distribution of which is smooth. Particularly, the homotopy of $\boldsymbol{H}$ can identified with, and thus defined as that mapping of $\boldsymbol{H}$ into $\mathcal{H}$ conserving the number of elements, i.e. the dimensionality $n$ of the vector between $\boldsymbol{H}$ and $\mathcal{H}$. If $\boldsymbol{H}$ is interpreted as the set of types on $\boldsymbol{C}$, this implies both "axiom 
of univalence" [@] and an (iso)morphism between the category of all categories and the pair of $\mathcal{H}$ and $\boldsymbol{H}$.

That consideration makes obvious the of the continuous (smooth) and discrete as one and the same well-ordering chosen as an ordinal among all well-orderings (permutations) of the same elements and it by itself accordingly. In other words, the continuous (smooth) seems to be class of equivalence of the elements of a set (including finite as a generalization of continuity as to finite sets).

Furthermore, the same consideration can ground (3) and (9) above, i.e. the way, in which a coherent state before measurement is equivalent of the statistical ensemble of measured states in quantum mechanics. The same property can be called "invariance to choice" including the invariance to the axiom of choice particularly.

This means that the pure possibility, e.g. that of pure existence in mathematics, also interpretable as subjective probability should be equated to the objective probability of the corresponding statistical ensemble once unitarity (energy conservation) has already equated $\mathcal{H}$ and $\boldsymbol{H}$.

Indeed, the set or its ordinal can be attributed to the elements of $\mathcal{H}$ and the statistical mix of all elements of $\boldsymbol{H}$ corresponding to a given element of $\mathcal{H}$. Any measurement ascribes randomly a certain element of the corresponding subset of $\boldsymbol{H}$ to any given element of $\mathcal{H}$. Thus measurement is not unitary, but a collapse of wave function.

Then, $\mathcal{H}$ and $\boldsymbol{H}$ can be interpreted as two identical but complementary dual spaces of the separable complex Hilbert space. Initarity means right their identity, and the nonunitarity of measurement representing a random choice means their complementarity.

That "invariance to choice" can ground both so-called Born probabilistic and Everett \& Wheeler "many-worlds" interpretations of quantum mechanics. The former means the probability for a state to be measured or a "world" to take place, and the former complement that consideration by the fact that all elements constituting the statistical ensemble can be consistently accepted as actually existing.

One can emphasize that the Born interpretation ascribes a physical meaning of the one component (namely the square of the module as probability) of any element of the field of complex numbers underlying both $\mathcal{H}$ and $\boldsymbol{H}$. After that, the physical meaning of the other component, the phase is even much more interesting. It should correspond to initarity, and then, it seems to be redundant, i.e. the field of real numbers would be sufficient, on the one hand, but furthermore, to time, well-ordering, and choice implied by it. In other words, just the phase is what is both physical and mathematical "carrier" and "atom" of the invariance of choice featuring the separable complex Hilbert space.

\section{ENTANGLEMENT AND THE ENTANGLEMENT VIEWPOINT TO CHEMICAL COMPOUND}

The definition of any quantum state (and thus system) as a point in the separable complex Hilbert space implies a fundamentally new option for two or more quantum systems to interact, in comparison with the case of classical mechanics and physics: entanglement.
Entanglement may be defined as the case where the separable complex Hilbert space of the total quantum system cannot be decomposed to a certain tensor product of the corresponding Hilbert spaces of the subsystems, or in other words, the latter spaces are not subspaces of the total space.

This means that the quantum whole cannot be represented as a product of its parts in the general case including that of entanglement. Thus entanglement is a corollary from that kind of holism featuring quantum mechanics.

Entanglement might not be defined in any real Hilbert space for it rests on the second dimension involved right by the complex Hilbert space. Entanglement may be furthermore equivalently defined as the non-unitary case right implied by involving the complex Hilbert space.

Particularly, the non-unitarity of entanglement means that the separation of conserving energy and conserving momentum is suspended: energy can be directly transformed in momentum and thus chemical energy into mechanical motion in the final analysis.

The corresponding quantity, which should be conserved in that generalizing case, is that of action. The quantity of action can be equated to that of (quantum) information directly for the sense of the fundamental Plank constant may be interpreted in thus. The case of conserving action (information) in comparison with that of energy and momentum separately can be visualized by that conservation, which is invariant to all space-time positions. For example, the conservation of action (information) implies the option of different times and their units.

Furthermore, if one conserves energy and momentum separately, this implies a certain space-time position (e.g. that of the Big Bang) to be postulated as universal.

The non-unitarity can be represented as a compound rotation of the constituent Hilbert spaces of the subsystems to each other axis by axis i.e. in any qubit internally if the two subspaces of the separable complex Hilbert space constituting any qubit are admitted not to be orthogonal to each other. Then, the corresponding qubit of the single total system will be the orthogonal equivalent of that entangled and thus nonorthogonal qubit.

One can visualize the comparison of the cases of entanglement and non-entanglement as curving as this is demonstrated by Figure @1.

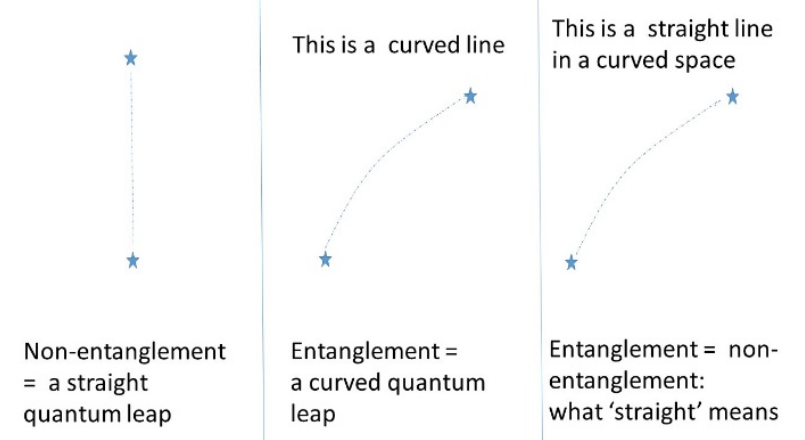

Fig.@1

The history of entanglement can be started since Neumann's Grundlagen der Quantenmechanik (1932), which (1) described mathematically rigorously the mathematical 
apparatus of quantum mechanics based on the separable complex Hilbert space, and (2) deduced the theorems about the absence of hidden variables in quantum mechanics on the same base. The latter implies the phenomena of entanglement in a sense. Indeed, the separability of the interacting quantum subsystems means the availability of hidden variables, and consequently their absence according to Neumann's theorem implies entanglement as the corresponding inseparability.

The explicit formulation of the entanglement problem should refer to 1935 and two papers:

Einstein, Podolsky, and Rosen's Can Quantum-Mechanical Description of Physical Reality Be Considered Complete? deduced the phenomena of entanglement from the mathematical apparatus of quantum mechanics, but considered as reductio ad absurdum for the completeness of quantum mechanics because of postulating the "elements of reality" as separable from each other.

Schrödinger's Die gegenwärtige Situation in der Quantenmechanik also forecast the phenomena of entanglement calling them "verschränkten Zustände". However, it unlike the former paper accepted their existence in reality.

The next main stage should be connected with Bell's On the Einstein Podolsky Rosen Paradox (1964), which demonstrates that the case of entanglement can be experimentally distinguished from that of its absent formulating a sufficient (but not necessary) condition of existence: the so-called violation of Bell's inequalities. It consists in the option for the correlations between two quantities in quantum mechanics to exceed the upper limit of correlation admissible in classical mechanics. That excess would confirm entanglement experimentally if a corresponding experiment is realized.

Kochen and Specker's paper The Problem of Hidden Variables in Quantum Mechanics (1968) generalized Neumann's theorem about the absence of hidden variables in quantum mechanics as to commuting quantities. It elucidated that the absence of hidden variables and therefore entanglement are due to wave-particle duality and to the invariance of the discrete and continuous (smooth) motion in the final analysis. Furthermore, the absence of hidden variables implies completeness and thus the phenomena of entanglement just for the Einstein - Podolsky - Rosen argument interpreted however as a confirmation rather than reductio ad absurdum. The invariance of the discrete and continuous in turn implies the equivalence of the standard and non-standard interpretation (in the sense of Robinson's analysis) and the axiom of choice, at last.

Information and quantum information particularly need fundamentally choice for both are quantities of choice measured in the units of elementary choice, correspondingly bits and qubits. Summarizing, the Kochen - Specker theorem is what founds information as the real and universal substance in quantum mechanics.

The Bell's inequalities were modified into a way more convenient for experimental tests in @ and soon the results of corresponding experiments were reported [@]. All of them have corroborated the existence of entanglement. A new both fundamental and applied area of research have thrived since the 90ies of $20^{\text {th }}$ century including: quantum computer, quantum communication, and quantum cryptography.
The special attention should be paid to the so-called backdoors problem. The mass of experiments confirming entanglement are indirectly and statistically admitting in principle alternative explanations without entanglement, namely "backdoors". If a certain backdoor is more or less probable as to a single experiment, that kind of explanation seems to be extremely improbable as to all corpus of those experiments. However, the reliable statistical methods to be unified different experiments as correlative to each other for their joint trustworthiness to be estimated are not yet elaborated.

In fact, entanglement implies a new paradigm not only in quantum mechanics and physics, but also in chemistry, biology, theory of information, astronomy, cosmology, mathematics and logic, and maybe etc. It has furthermore direct reflections in metaphysics and all branches of philosophy and even in theology. Thus, it implies a fundamental turn in human knowledge. The proofs for that should be more than convincing for the reorganization and restructuring would take much time, efforts, and resources. Furthermore, they would need even the alternation of generations for the change of viewpoints would be often impossible for the individuals. The thousands of present investigations would turn out to be outdated and even meaningless. For example, even maybe the biggest contemporary scientific project, GAC - CERN would turn out to be outdated needing fundamental reinterpretation.

As a result, entanglement and the corresponding theory of quantum information continue not to be officially recognized, particularly not confirmed by any Nobel prize though not refuted. They are the one alternative of the most fundamental scientific dilemma nowadays. Meanwhile, the scientific research continues in the old track though the scientific research in the new one increases. So, the state of affairs in science as to quantum information can be called ambiguous.

The usual viewpoint to chemical substance and particularly to chemical compound considers it as a collection of molecules of a single kind containing really some impurities which should be insignificant amounts, which are molecules or atoms of other chemical substances. The carrier of all chemical properties of a compound is its molecule even as a single one. Chemical compounds can be obtained by chemical reactions, after which the initial ingredients, which furthermore are included in the ultimate compound are linked by chemical bonds, which represent pairs of electrons.

The chemical compounds are constituted by atoms of the elements of the Periodic table interacting between their electron shells. The shells are ordered in a series of layers (strata), from which chemically most active is the last one, which is incomplete in general. If it is complete as a particular case, the corresponding chemical elements are exceptionally slightly chemically active: "inert" ("noble").

The number of electrons in the shell corresponds to its number in the Periodic table, and the number of electrons in the last, chemically active layer to the valence (@valency) or the group, to which a certain chemical element belongs. The elements of a certain chemical group have similar chemical properties, which are essentially different from those of the other groups. The difference between the numbers of the groups (the valences) is bigger, the difference in the properties is more essential. 
Those rules are made further more precise and complemented, many cases of exceptions are described and explained.

The chemistry of a single element, namely carbon, is isolated as "organic chemistry" as far as carbon is able to constitute very complex, long, and branched molecules, being furthermore the base of life.

However, the viewpoint in the present paper explaining chemical substance and compound by the corresponding wave functions and their entanglement both generalizes the "classical" viewpoint in chemistry and changes its gestalt and thus essence.

After generalizing, it comprises as generalized chemical compounds all cases of entangled wave functions, i.e. practically all physics of elementary particles.

After changing the gestalt, the essence of chemical compound is grounded on entanglement, and its new properties are explained by it fundamentally. The chemical bond as well as the ability of that is reduced to the way of pinning two or more wave functions to be stably entangled.

The main problem is how entanglement is able to change the properties of any chemical substance immediately.

The base of explanation consists in the equivalent reinterpretation of an entangled composed quantum system as a single, and total but reordered system. Then, two fundamental kinds of reordering after entanglement would be possible: (1) finite (or quantitative), and (2) transfinite or qualitative. As far as the separable complex Hilbert space can be considered as a transfinite set or series of qubits, the option (2) is both admissible and quantitative way for any qualitative change in the properties to be represented.

That equivalence can be demonstrated as follows. One considers one single qubit referred to two entangled wave functions. Then, a "normal", i.e. non-entangled qubit is juxtaposed to the former one. The values of probability in the corresponding point of the probability will be changed in a jump-like way in general. One can restore the continuity and even smoothness of both corresponding probability distributions if the qubit at issue is reordered into a new place under the condition for both probability distributions to be smooth. This is always possible under the condition for transfinite reordering to be admissible.

In other words, a single probability distribution can be adjusted to be smooth if the corresponding point is reordered in a new relevant place in the arbitrarily long finite series, i.e. by a finite reordering. The other probability distribution can be adjusted only choosing a relevant transfinite segment of a certain other arbitrarily long finite series The quantitative change needs one dimension, and the qualitative change means another dimension thus implying trans-finiteness (infinity) to be consistently describable to the former dimension of finiteness.

The statement can be proved by induction for an arbitrary number of entangled functions after it is proved for two ones as above.

Then, a single transfinite wave function, consisting of an arbitrary number of arbitrarily long subseries of qubits, can represent exhaustedly any chemical substance and all its properties whether chemical or physical, or whatever else. Any quality and thus property of the chemical substance would correspond to a certain arbitrarily long series of qubits, and thus to the case (2) of reordering. The case (1) of reordering would be that of an arbitrary (jump-like in general) change of the value of a certain quantity.

Physics as a rule studies rather (1) unlike chemistry researching rather (2). Thus, the entanglement approach to chemical substance tends to unify physics and chemistry on the base of quantum mechanics and especially quantum information, but to distinguish them according to the viewpoint to substance: rather quantitative in physics vs. rather qualitative in chemistry. The base of that distinction is the separation of all finite re-orderings vs. all transfinite ones due to entanglement of a few wave functions.

However, that last mentioned separation is fussy once the separable complex Hilbert space is what is meant and utilized.

(1) Indeed, any infinite or transfinite series admits a oneto-one mapping to some (but fundamentally unknown) finite set as a matter of pure existence if both axiom of choice (in set theory) and axiom of induction (in Peano arithmetic) are valid.

(2) Furthermore, one may represent that "pure existence" (of some fundamentally unknown finite set being an equivalent of any certain infinite set for a one-to-one mapping) as a probability distribution on all finite sets to be that equivalent. A characteristic function can be always assigned to the above probability distribution.

(3) Then the change from an infinite set to another can be always represented as a wave function, i.e. as a point in the separable complex Hilbert space for that other infinite set can be also represented as another probability distribution on the same set and consequently as another independent (and thus orthogonal) characteristic function, which together with the former one represents right the wave function is questions.

The three considerations together imply that the separable complex Hilbert space is just that structure, which is able to unify all finite and infinite re-orderings and thus the viewpoint of physics and chemistry to substance.

Particularly, the same statement implies that the change of the properties including the properly chemical ones of any chemical compound in comparison to its ingredients is probabilistic as to a single molecule (or atom) of it. The really observed stability of the changed properties of the chemical compound should be due to the huge number of molecules (or atoms) of chemical substance generating a certain, rigorously determined way of de-coherence of all entangled wave functions of the chemical substance at issue. Thus, just one property turns out to be always chosen among the hypothetical veer of possible properties assignable to a single molecule (atom) of chemical substance.

As to the generalized chemical substances and compounds (such as elementary particles), they are observed as individuals for both their frequent instability and individual registration of the measuring apparatuses.

\section{AN ENTANGLEMENT THEORY OF CATALIC ACTION AND THERMODYMANIC CONDITIONS OF OD CHEMICAL REACTION}

The chemical reactions are influenced rather "mystically" by two groups of factors: (1) impurities and catalysts, and (2) the 
thermodynamic conditions of the reactions. Both seem to act chemically without being chemical in nature. The impurities turn out not to be changed after the end of the reaction. One can suggest that they form some intermediate sub-reactions, which facilitates or prevent the total reaction, or act indirectly by changing (2). The thermodynamic conditions themselves can be reduced to (quantum) mechanics of atoms or molecules according to (quantum) statistical thermodynamics. The mechanism, in which (quantum) mechanical motion influence the result of the reaction, is not clear enough. It needs certain elucidation how chemical energy due to the appearance or decay of chemical bonds can be directly transformed into the mechanical motion of atoms or molecules.

The entanglement theory of chemical compound and reaction meant in the present paper is able to explain both;

(2) The thermodynamic conditions of the chemical reaction are understood by means of the total conservation of energymomentum in entanglement rather than energy and momentum separately. Those two separate conservations correspond to unitarity in the "classical" quantum mechanics and to the state of zero entanglement between the subsystems of any quantum system. Then, energy is not able to transform in momentum directly, or vice versa. The influence of thermodynamic conditions on chemical reaction seem to be really "mystical" in that framework admitting only quasiexplanations e.g. of how the bigger velocity of atoms or molecules assists their approaching and thus the appearance of chemical bonds.

Entanglement is defined to be non-unitary, and thus conserves jointly energy and momentum, i.e. energymomentum $^{9}$ as a whole. Then, this can involve a mechanism of mutual direct transformation of chemical energy and mechanical motion. Indeed, chemical energy is the unitary energy of the chemical compound as a whole, and it be represented equivalently as a certain degree of entanglement, i.e. a complicated rotation what non-unitarity is. That degree of entanglement corresponds unambiguously to a certain relative mechanical motion of the entangled subsystem. That is way for chemical energy to be transformed directly into mechanical motion. The same way seen reversely explains how mechanical motion is able to transform into chemical energy.

The essence of that transformation explained by entanglement rests on the invariance to two viewpoints: the chemical compound seen a single whole and thus as a single unitary (i.e. non-entangled) wave function and as composed by entangled subsystem. Then, the entangled subsystems can be equivalently represented as moving mechanically relatively to each other.

(1) The influence of impurities and catalysts as the particular case of strong and intended influence can be explained by entanglement including or not the appearance of partial or intermediate chemical compounds, in which the impurities participate or not.

The criterion for a chemical substance to be available is the chemical bond though short-time. It serves to pin two or more wave functions and thus to stabilize their entangled wave

\footnotetext{
${ }^{9}$ General relativity conserves also energy-momentum and this is not a random fact. It is explained in Section 5 and 6.
}

function. However, this is a sufficient, but not necessary condition for an impurity to influence a chemical reaction.

For example, two wave functions can interact by entanglement without the pinning and stabilizing chemical bond, but in virtue of their spatial neighbourhood. Indeed, the effect is probably short-time, but this can be enough as an intermediate stage for interacting with a third wave function, after which that of the impurity turns out to be detangled and thus able to assist the ultimate entanglement of new and new atoms and molecules to the chemical compound. Particularly, short-time chemical bonds can appear between the impurity and some of the ingredients of the ultimate, i.e. stable chemical compound.

\section{A GENERALIZED CONCEPT OF CHEMICAL COMPOUND COMPRISING ALL "ELEMENTARY PAPRTICLES"}

The prejudice distinguishes the strong, electromagnetic, and weak interactions from each other for they are so different in strength, range, and effects. Thus, the Standard model unifying them in a sense ${ }^{10}$ and adding the Higgs boson to explain how the rest masses appear seems to be so dramatically creative as to common sense.

However, if one "brackets" the above obvious differences and unifies them as forms of conservation, i.e. symmetries of the separable complex Hilbert space, opposing them only to the asymmetries, a quite unexpected viewpoint is outlined, but relative to that of meta-chemistry:

The asymmetries correspond to both entanglement and non-self-adjoint operators, and the symmetries to the zeroentanglement, chemical bonds in a generalized sense, and self-adjoint operators.

Thus any chemical substance of that generalized sense share which is not decomposable as to the chemical elements, which turn to be rather those of the Standard model than of the Periodic table. The chemical compounds are an enormous number including not only those in the "old" chemistry, but furthermore all the rest elementary particles of physics \& elements of the "old" chemistry.

The classical chemical compounds are based only to the electromagnetic interaction of the electron shells of atoms or molecules. The new viewpoint accepts that property rather as accidental, and the essential definition of "chemical compound' rests on the entanglement of constituting wave functions. Indeed, that entanglement needs some kind of conservation as a "carrier" to be stable in space-time. The most of the "elementary particles" are too unstable. This means that the carrier is too restricted in space-time and the corresponding entanglement decays. Only confinement and electromagnetic interaction are able to be stable carriers of entanglement.

Anyway, the fact of entanglement rather than its stability should be leading if one defines 'chemical compound'.

Then, three basic kinds of 'chemical compound' after its generalization can be distinguished according to the three

\footnotetext{
${ }^{10}$ It unifies properly only the weak and electromagnetic interactions and adds an analogy between the strong and electro-weak interactions.
} 
fundamental interaction in the Standard model as kinds of carrier of entanglement.

Furthermore, the concept "carrier of entanglement" needs to be additionally elucidated. Its essence means a certain reference frame of zero entanglement to be somehow determined so that entanglement is able to make sense.

Further, zero entanglement is natural to be defined as the independence of conjugates. If the quantity of action is constant in a sense (e.g. minimal as in the principle of least action) both conjugates are also constant in the sense of Emmy Noether's theorems (1918). Then, the constancy of the one can be interpreted as symmetry, and that of the other as conservation due to the former symmetry.

The distinction of symmetry and conservation is not essential for they can be swapped. The concept of symmetry originates from geometry and the geometric representation of space-time, and that of conservation from physics and the laws of conservation in it. However, physics and geometry turn out to be rather indistinguishable from each other in quantum mechanics ${ }^{11}$ for the separable complex Hilbert space.

Thus zero entanglement can be interpreted philosophically as physics and geometry independent of each other, though indistinguishable. That viewpoint can be reversely seen:

The chemical bonds as zero entanglement and representing admissible kinds of conservation means furthermore a privileged point in the corresponding relevant space defined as its symmetry. Indeed, if physical conservation is interpreted as a kind of symmetry, geometric symmetry in turn is able to be interpreted as a kind of conservation, i.e. a point in the corresponding space to be chosen as privileged.

As a conclusive statement, the chemical bonds can be interpreted in the final analysis as a privileged reference frame, that of "rest", to which both mechanical motion and entanglement make sense or an absolute, non-relative sense.

Possible applicants for that privileged reference frame might be e.g. that of the Big Bang, of our position in the universe, or that implicitly defined by the Standard model after explicitly defining what is conserved: namely, the wave functions of its "elements" for the wave functions of them are not entangled, and they are just elements. One too speculative, but rather elegant hypothesis would explore the consequences if some of the three naturally privileged reference frames, mentioned above, are presupposed to coincide.

\section{CONCLUSIONS ABOUT "TRIGGER FIELD"}

The "Trigger field" seems to be very well interpretable as entanglement and thus as a field of quantum information, which means the direct interaction of two or more quantum field after 'quantum field' is defined as any mapping of spacetime into the separable complex Hilbert space.

Thus quantum field can be in turn identified as a field of quantum information for Minkowsky space as space-time according to special relativity can be considered as the nonstandard interpretation of that Hilbert space as the axiom

\footnotetext{
${ }^{11}$ They are indistinguishable also in general relativity, the other physical theory as fundamental as quantum mecanics.
}

of choice is admitted ${ }^{12}$. For the initial complex Hilbert space is postulated as separable, quantum field as a field of quantum information is isomorphic to a mapping of the separable complex Hilbert space into itself.

Thus any operator either self-adjoint or not can be equated to quantum (information) field. All admissible physical quantities being defined as self-adjoint operators in quantum mechanics correspond to the quite reasonable restriction to be excluded any intertemporal interactions therefore violating causality from the set of acceptable physical quantities. However, entanglement being right the corresponding generalizing case corresponds just to that kind of forbidden physical quantities violating causality. Anyway causality can be secondarily restored if a universal force field such as the gravitational one be introduced.

Indeed, any force and then force field means right restoring causality by adding in the causal picture that 'force' swapping the causal order as independent of it and acting on it transforming it into another. Then, that universal gravitational field should represent isomorphically rather that only equivalently entanglement in an acceptable, i.e. causal way.

Until now in this paper, entanglement has identified as properly the general case of chemical interaction generating the new properties of any chemical compound in a broad sense. Indeed, all chemical substances including compounds are considered as usual "outside" and thus as a whole, the internal space-time structure of which can be "bracketed" for space-time is not a chemical concept inherently. Gravity as well the missing "dark" matter and energy should be allocated in that hidden space-time structure of all chemical compounds in a broad sense.

The "forbidden" intertemporal swaps within the mapping of the complex Hilbert space, i.e. seen internally, being right entanglement, are expressed externally as the change of the properties of the compound at issue in comparison with its ingredients. Thus entanglement unifies chemical and qualitative viewpoint "outside" with the physical and quantitative one "inside".

Indeed, one reading "The Trigger" can assure that the initial direction of research led to the Trigger effect to be found is that of creating "gravitational laser". It right turns out to be that emitter of quantum (information) field able to transform any chemical substance into another directly, i.e. without the action of any other interacting chemical substance.

The mechanism and structure of that emitter are not explicitly described in the novel, however one can suggest them as far as they should cause the "Trigger effect" in question, which namely follows.

The fundamental Planck constant may be interpreted as equating the quantity of (quantum) information to that of action. Consequently, the direct physical influence able to cause a certain chemical transformation should have the physical dimension of action.

Then, if one concentrates a constant energy-momentum emission such as that of a usual laser in a certain and absolutely exactly determined space-time point, an equivalent value of quantum information will turn out to be generated,

\footnotetext{
${ }^{12}$ Particularly, "separable" in the definition of the complex Hilbert space is not necessary already for the axiom of choice transforms any Hilbert space into separable.
} 
but valid only to this space-time point and different in any other one.

Further, one can utilize a set of an enough number of lasers able to model exactly enough the wave function of a certain chemical substance, the interaction with which is intended to be simulated.

At last, a certain chemical substance interacting with that simulated by the above set of lasers should be allocated in the cross-point of all lasers, and they switched on.

If a certain chemical compound appears there, being indistinguishable from the real one, this will be an experimental witness of a series of fundamental and exceptionally important hypotheses, and first of all:

(1) Quantum information is the substance of the world.

(2) Chemical interaction is entanglement.

Any chemical substance can be created rather than only modeled or simulated by purely physical instruments such as lasers.

Furthermore, the available chemical substance in the crosspoint of all lasers can be not less indistinguishably replaced by a second set of lasers corresponding to its wave function.

\section{PHILOSOPHICAL, METAPHYSICAL, AND METHODOLOGICAL INTERPRETATIONS}

The usual prejudice to chemistry is that it is a kind of applied physics without any essential fundamental meaning for the understanding of the world nowadays. Its link to mathematics is entirely mediated by physics.

That prejudice is inconsistent to the present viewpoint. By reformulating and generalizing chemistry by quantum information and entanglement, it turns out to be the fundamental cognition and thus viewpoint to the world including physics as the quite particular, but rather important case of "pure chemical elements" as what the elements of the Standard model are also included rather than those of Periodic table as in the "old" chemistry, that in a narrow or classical sense.

The base for that revolution in metaphysics, which should be called rather meta-chemistry already than metaphysics, is the reinterpretation of chemical compound as a direct quantum information interaction of wave functions pinned to each other in a certain (very tiny) space-time area resulting into a single entangled wave function of the chemical compound. The chemical bonds such as between electron shells in the old chemistry are necessary only for pinning in space-time of the constituting wave function to be stable entangled there, but they are not either carriers or cause of the new and possible absolutely different properties of the chemical compound. Anyway, the shared (therefore maximally entangled) wave function of the bond influences essentially but not only to the properties of the compound.

Thus, chemistry and mathematics turn out to be in turn "entangled" by entanglement removing physics as a redundant and even harmful mediator. That new meta-chemistry tends to be underlain by a mathematical universum of information or quantum information therefore facilitating certain neoPythagorean viewpoints.
That fundamental physics reinterpreted in the framework of meta-chemistry repay attention from the symmetries (and the corresponding conservations) to wave function, which is not symmetric in general, and to entanglement as a direct interaction of arbitrary wave functions rather than symmetries shared in the separable complex Hilbert space therefore shared by independent (and thus non-entangled) wave functions.

Consequently, that refocused physics changes the most fundamental concept of physical interaction from symmetry and conservation of independent quantities to the quantuminformation dependence and thus interaction, i.e. to chemical reaction in the new generalized sense considering the old chemistry as a very important, but anyway quite particular case. The symmetries themselves, then, mean both space-time pole of maximal entanglement and bond able to pin stably the constituting wave functions to each other.

The missing dark energy and matter should be chemical, i.e. due to entanglement. The non-dark matter and energy, i.e. the classical ones being those of symmetries are the particular case of zero entanglement, for "symmetry" means just the absence of entanglement, and vice versa: the availability of entanglement instantly violates the symmetry. They are "dark" only for the wrong bounds of our knowledge excluding entanglement and quantum information from the mainstream (or "paradigm") of physical cognition. Dark matter and energy are everywhere, but visible only from the viewpoint of the new Pythagorean meta-chemistry. They are chemical in their essence in that generalized sense of chemistry meaning entanglement.

\section{SUMMARY, CONCLUSIONS \& FUTURE WORK}

Clark and Kube-McDowell's sci-fi idea about the "Trigger field" seems not to contradict the contemporary knowledge in chemistry and physics and consequently certain real analoga of it might be ever constructed if the huge technical obstacles be overcome. Anyway new cognition might also refute that option in principle as contradicting some unknown yet law of nature.

Even much more that only consistency, that idea is able to change the total gestalt of our knowledge from metaphysics to a new, rather mathematical kind of meta-chemistry based on the concept of entanglement as a direct (quantum) informational interaction underlying any energetic entity properly studied by physics.

Then, the concept of chemical compound should be generalized as any direct interaction of wave functions more or less stable in a certain space-time area, mainly due to the bonds of conservation of three physical interactions: strong, weak and electromagnetic, and nonlinearly resulting into the new entangled function of the compound. That generalization would consider the "elementary particles" as chemical compounds as well rather than only the "classical" chemical compounds being aggregates of atoms and due to electromagnetic interaction and electron bonds of their shells.

The new properties of the compound in comparison with its ingredients is a fundamental, but inexplicable fact in the framework of the "old" chemistry concentrated on chemical bond rather than to the essential and stable interaction of wave 
function caused by those bonds. The meaning of the bonds is only to pin the wave functions in a certain space-time area for their entangled wave function to be stable there.

Electromagnetic interaction being both strong enough and unlimited in range is especially suitable for chemical electron bonds able to generate the complete variety of classical chemical compounds. However, the bonds being rather constructive than functional elements cannot explain why and how the properties of the compound are changed to those of the ingredients.

Entanglement is what elucidates and can describe quantitatively the way of change of the properties of the ingredients into the compound. It can be generally represented as an arbitrary operator on the complex Hilbert space and thus as processing quantum information. Entanglement being identified as the properly chemical interaction should include the particular cases of zero entanglement what are the fundamental physical interaction recognised until now in the Standard model and describable by self-adjoint operators. Gravity might not be among them, but being attachable as the physical appearance of nonzero entanglement as energymomentum.

The dark matter and energy revealing themselves in gravitational phenomena of mega-scale are right the difference between the energy-momentum due to nonzero entanglement to the visible (for the contemporary knowledge) matter and energy of the above standard interactions of zero entanglement and thus conserving energy and momentum separately (unlike general relativity). Thus one can say that the dark matter and energy being everywhere together with the visible one are chemical in that generalized sense, to which the paper is devoted. They are invisible only for the identification of physical interaction with unitarity as conservation and thus with zero entanglement.

The way how entanglement change the properties can be call transfinite nonlinearity and described "from bird's eye view" as follows:

One can postulate a well-ordering of any statistical ensemble, due to the smoothness of the corresponding probability distribution. This means that neighbouring states or elements should be almost equally probable. Then, entanglement causing an arbitrary probability change of any argument of the probability distribution will violate therefore arbitrarily that smoothness. It can be restored and equivalently interpreted as the smoothness of another well-ordering reordered according to the above rule: neighbouring arguments are almost equally probable.

Furthermore, that reordering should comprise two main cases: (1) where the change of the position of a certain argument of the probability distribution after reordering is a finite ordinal, and (2) where that is a transfinite ordinal.

The case (1) should be ascribed to a single quantity, but (2) to two different quantities and thus to change of quality, or physical dimension. The existence of (2) is right experimentally proved by the change of properties in any chemical compound in comparison to its ingredients.

Indeed, one can assign a single wave function exhaustedly describing any chemical substance. That function will have as many finite fragments and corresponding dimensional gaps between them as many qualities includes its properties. Reordering, which is trans-dimensional, i.e. transferring a certain argument between different fragments, being due to entanglement, furthermore will change a certain quality and thus property.

That mechanism can simply explain the influence of catalyst or any impurity in general remaining unchanged in the course of the corresponding chemical reaction as well as the significance of tiny concentrations of catalysts or impurities. The catalysts and impurities are able to modify the wave functions of certain ingredients unstably without chemical bonds only for the space-time neighbourhood of molecules or atoms. That modification facilitates or prevent from the interaction of the wave functions constituting the compound, but the catalysts or impurities remain unchanged and able to modify the wave functions of new and new atoms or molecules.

The chemical interaction based on entanglement allows of elucidating the way, in which the chemical energy due to the change of wave function is able to be transformed into the thermodynamic quantities of huge statistical ensembles of atoms or molecules, being mechanical in a classical sense, and vice versa: why the thermodynamic condition of chemical reaction influence essentially on both course and even realization of it:

Entanglement conserves energy-momentum rather than energy and momentum separately. Thus it offers the mechanism, in which chemical energy can be transformed in mechanical motion directly without the mediator of any thermodynamic engine, which is not really available in any chemical reaction.

The idea of Trigger field seems not to contradict physical laws if it is interpreted as entanglement. Entanglement can be interpreted as the field of both physical action and quantum information for the fundamental Planck constant allowing of them to be equated. The simplest way of constant action corresponds to constant energy-momentum in a certain spacetime point. That most elementary case corresponds to the idea of quantum field ascribing a wave function to any space-time point and thus a certain value of action in it.

The constant entanglement in a space-time point is realized by the chemical bond pinning two or more arbitrary wave functions to each other stably, which generate a single and stable entangled wave function, that of the compound at issue. The necessary space-time constancy besides chemical bonds can be furthermore realized as the cross-point of a sufficient number of "information lasers". Those "information lasers" can be usual lasers chosen in way for the energy-momentum of each of them to correspond absolutely exactly to all independent axes of the wave function of a certain chemical substance and thus to model it in a quantum-information mode.

If that set of information lasers is directed to a certain chemical substance in an absolutely exactly certain space-time point, a corresponding entangled function should appear just there for the exact cross-point is a perfect equivalent of the chemical bond pinning the wave functions. Consequently, the chemical substance available there should transform into the corresponding chemical compound under the action of the information lasers modelling the other chemical substance(s). This describes an implementation of "Trigger field" as well as an experimental way to be checked its existence in reality. 
Furthermore, the available chemical substance should be able to be substituted by another set of information lasers modeling it. The entangled wave function of the compound should appear in the cross-point again as well. That entangled wave function should behavior indistinguishable from the corresponding real compound.

That kind of thought experiments, which might ever be realized as real, proves or would experimentally prove that any chemical substance is not any other than an (entangled) wave function and thus quantum information.

Quantum information as the substance of the world would be confirmed revealing new immense areas for human cognition.

\section{REFERENCES}

[1] Bader, R. F. W. B. Atoms in Molecules: A Quantum Theory. Oxford: Clarendon Press (1994).

[2] Primas, H. Chemistry, Quantum Mechanics and Reductionism: Perspectives in Theoretical Chemistry. Heidelberg, Berlin: Springer (1981).

[3] Özdin, N. et al. Chemistry The History of Atom, The Periodic Table and Radioactivity. Web: Zambak Publishing (2008).

[4] Pasachoff, N. Marie Curie: And the Science of Radioactivity. Oxford - New York: Oxford University Press (1996)

[5] Malley, M. C. Radioactivity: A History of a Mysterious Science. Oxford - New York: Oxford University Press (2011)

[6] Gavroglu, K., Simoes, A. Neither Physics nor Chemistry: A History of Quantum Chemistry. Cambridge, MA; London, England: MIT Press (2012).

[7] Cooksy, A, Physical Chemistry: Quantum Chemistry and Molecular Interactions. Boston, etc.: Prentice Hall (2013).

[8] Pauling, L. The Nature of the Chemical Bond. Ithaca, NY: Cornell University Press; Oxford: University Press (1960).

[9] Ōki, M. Chemistry of Rotational Isomers. Heidelberg - Berlin: Springer (1993).

[10] Prud'homme, R. Flows and chemical reactions (Handbook). London: ISTE Ltd.; Hoboken, NJ: John Wiley \& Sons, (2012).

[11] Holtz, P. O., Qing Xiang Zhao. Impurities Confined in Quantum Structures. Berlin, Heidelberg: Springer (1999).

[12] Sowa Jr, J. R. Catalysis of Organic Reactions. Boca Raton, FL, etc.: Taylor \& Francis Group: CRS Press (2005).

[13] Lew, K. Chemical Reactions (Essential Chemistry). New York: Chelsea House Publications (2008).

[14] Zaikov G. E., Kozłowski, R. Chemical reactions in gas, liquid, and solid phases: synthesis, properties, and application. New York: Nova Science Publishers (2010).

[15] Wyatt, R. E., Zhang, J. Z. H. Dynamics of molecules and chemical reactions. New York: Marcel Dekker (1996).

[16] Luis, S. V., Garcia-Verdugo, E. Chemical reactions and processes under flow conditions. Cambridge: Royal Society of Chemistry (2010)

[17] Sidis, V. Some Recent Developments in the Molecular Treatment

of Atom-Atom collisions. In: Duadel, R et al (eds.) Quantum Theory of Chemical reactions I. Collision Theory, Reaction Path, Static Indices. Dordrecht, etc.: Reidel (1979), pp. 1-23.

[18] G. Leroy, G. et al. Contribution to the Theoretical Study of Reaction Mechanisms. In: Duadel, R et al (eds.) Quantum Theory of Chemical reactions I. Collision Theory, Reaction Path, Static Indices. Dordrecht, etc.: Reidel (1979), pp. 91-143.

[19] Gavezzotti, A., Simonetta, M. Structure and Reactivity: An Extended Huckel Approach. In: Duadel, R et al (eds.) Quantum Theory of Chemical reactions I. Collision Theory, Reaction Path, Static Indices. Dordrecht, etc.: Reidel (1979), pp. 145-159.

[20] Germain, J. E. Theoretical Background of Heterogeneous Catalysis. In: Duadel, R et al (eds.) Quantum Theory of Chemical reactions III. III. Chemisorption, Catalysis, Biochemical Reactions. Dordrecht, etc.: Reidel (1980), pp. 1-13,

[21] Coletti, C., Billing, G. D. Quantum dressed classical mechanics: application to chemical reactions. Chemical Physics Letters 342(1-2): 65-74 (1987).

[22] Eyring, H. Quantum Mechanics and Chemical Reactions. Chemical Reviews 10(1): 103-123 (1932).

[23] Zhang, Y., Berman, G. P. Kais, S. The radical pair mechanism and the avian chemical compass: Quantum coherence and entanglement. International Journal of Quantum Chemistry 115(1): 1327-1341 (2015).

[24] Trout, C. J., Brown, K. R. Magic state distillation and gate compilation in quantum algorithms for quantum chemistry. International Journal of Quantum Chemistry 115(19):1296-1304 (2015). 
[25] Szalay, S. et al. Tensor product methods and entanglement optimization for ab initio quantum chemistry. International Journal of Quantum Chemistry 115(19): 1342-1391 (2015).

[26] Sjöqvist, E. Geometric phases in quantum information. International Journal of Quantum Chemistry 115(19): 1311-1326 (2015).

[27] Kais, S. Introduction to Quantum Information and Computation for Chemistry. In: Kais, S., Rice, S. A., Dinner, A. R. (eds.) Quantum Information and Computation for Chemistry (Advances in Chemical Physics 154) Hoboken, NJ: John Wiley \& Sons (2014), pp. 1-38.

[28] Love, P. J. Back to the Future: A Roadmap for Quantum Simulation from vintage quantum chemistry. In: Kais, S., Rice, S. A., Dinner, A. R. (eds.) Quantum Information and Computation for Chemistry (Advances in Chemical Physics 154) Hoboken, NJ: John Wiley \& Sons (2014), pp. 39-66.

[29] Kedzeira, D., Kaczmarek-Kedzeira, A, Remarks on Wave Function Theory and Methods. In: Leszczynski, J. Handbook of Computational Chemistry. Dordrecht: Springer (2014), pp 55-93

[30] Helgaker, T. et al. Wave Function Based Quantum Chemistry. In: Bultinck. P. et al. (eds) Computational Medicinal Chemistry for Drug Discovery. New York - Basel: Marcel Dekker, pp. 57-87.

[31] Gregory J. O. et al. Unrestricted Perfect Pairing: The Simplest Wave-Function-Based Model Chemistry beyond Mean Field. Journal of Physical Chemistry A 109(40): 9183-9192 (2005).

[32] Kutzelnigg, W. Theory of electron correlation. In: Rychlewski, J. (ed.) Explicitly Correlated Wave Functions in Chemistry and Physics Theory and Applications. Dordrecht: Springer (2003), pp. 3-90.

[33] Heidegger, M. Vom Wesen der Wahrheit. In: Wegmakrken. Frankfurt AM: Klosterman, 177-201 (1976)

[34] Heidegger, M. Erläuterungen zu Hölderlins Dichtung. Frankfurt AM: Klostermann (1951)

[35] Derrida, J. L'écriture et la difference. Paris: Seuil (1967).

[36] Derrida, J. De la grammatologie. Paris: Minuit (1967).

[37] Ricœur, P. La métaphore vive. Paris: Seuil (1975).

[38] Schrödinger, E. Die gegenwärtige situation in der Quantenmechanik. Die Naturwissenschaften 23(48): 807-812; 23(49): 823-828; 23(50): 844-849 (1935).

[39] Sartre, J.-P. L'existentialisme est un humanism. Paris: Nagel (1946).

[40] Heidegger, M. Identität und Differenz. Pfullingen: Günter Neske (1957).

[41] Seeburger, F. F. (1975) Heidegger and the Phenomenological Reduction. Philosophy and Phenomenological Research 36(2): 212-221 (1975)

[42] Morrison, J. C. Husserl and Brentano on Intentionality. Philosophy and Phenomenological Research 31(1): 27-46 (1970).

[43] Husserl, E. Cartesianische Mediationen (1929). Cartesianische Meditationen Und Pariser Vorträge. Husserliana. Bd. 1. Den Haag: M. Nijhof (1950).

[44] MacDonald, P. S. Descartes and Husserl. The Philosophical Project of Radical Beginnings. Albany: State University of New York Press (2000).

[45] Ricœur, P. Husserl: An Analysis of His Phenomenology (Northwestern University Studies in Phenomenology and Existential Philosophy). Evanston: Northwestern University Press, (1967).

[46] Henry, M. C'est moi la Vérité. Pour une philosophie du christianisme. Paris: Seuil (1996).

[47] Marion, J.-L. Dieu sans l'être. Paris: Fayard (1982).

[48] Overgaard, S. Epoché and Solipsistic Reduction. Husserl Studies 18 (3): 209-222 (2002).

[49] Mahnke, D. From Hilbert to Husserl: First Introduction to Phenomenology, Especially That of Formal Mathematics. Studies in History and Philosophy of Science (Part A) 8 (1): 71-84 (1977).

[50] Tieszen, R. L. Mathematical Intuition: Phenomenology and Mathematical Knowledge. Dordrecht, etc.: Kluwer Academic, (1989).
[51] Seidel, G. J. Martin Heidegger and the pre-Socratics: an introduction to his thought. Lincoln, NE: University of Nebraska Press (1964).

[52] Iamblichus. The life pf Pythagoras. Krotona - Hollywood, CA: Theosophical Publishing House (1918).

[53] Gorham, G., Hill, B., and Slovwik. E. The language of nature. Reassessing the mathematization of natural philosophy in the seventeenth century. Minneapolis: University of Minnesota Press (2016).

[54] Hardy, L. Phenomenology of natural science (Series: Contributions to phenomenology, v. 9) Dordrecht; Boston: Kluwer Academic Publishers (1992).

[55] Aukstuolyte, N. The Interrelationship between the Mathematization of Science and the Idea of Universal Formal Language of Science. European Scientific Journal 10(12): 442452 (2014).

[56] Wilner, J. The Mathematization of Nature: Galileo, Husserl, Mandelbrot. In The Yearbook of Comparative Literature 58: 235240 (2012).

[57] Quine, W. V. Success and Limits of Mathematization. In: Der 16. Weltkongress für Philosophie Volume 1: 439-446 (1983).

[58] Pawlowski, T. The Limits of Measurement and of Mathematization in the Social Sciences. In: Der 16. Weltkongress für Philosophie Volume 2: 439-446 (1983).

[59] Lenhard, J., Otte, M. Two Types of Mathematization. Philosophical Perspectives on Mathematical Practice (Texts in philosophy, 12) London: College Publications, 1018-1025 (2010).

[60] Apostolatos, N. Informatics and the Internal Necessity for the Mathematization of the Sciences. In: Applied Mathematics and Parallel Computing. Heidelberg: Physica, 1-10 (1996).

[61] Roux, S. Forms of Mathematization (14th -17th Centuries). Early Science and Medicine 15(4/5): (2010), pp. 319-337 (2010)

[62] Monteiro, S The Search for the Mathematization of the Social Disciplines. In: The Application of Mathematics to the Sciences of Nature: Critical Moments and Aspects. New York: Springer, 245252 (2002).

[63] Born, M. Physical Aspects of Quantum Mechanics. Nature 119 (2992): 354-357 (1927)

[64] Conway, J., S. Kochen. The Free Will Theorem. Foundations of Physics 36(10): 1441-1473 (2006).

[65] Conway, J., S. Kochen. The Strong Free Will Theorem. Notices of the American Mathematical Society 56(2): 226-232 (2009).

[66] Neumann, J. Mathematische Grundlagen der Quantenmechanik. Berlin: Springer, 167-173 (1932).

[67] Kochen, S. and Specker, E. The Problem of Hidden Variables in Qunatum Mechanics. Journal of Mathematics and Mechanics 17(1): 59-87 (1968).

[68] Husserl, E. Die Krisis der europäischen Wissenschaften und die transcendentale Phänomenologie: Eine Einleitung in die phänomenologische Philosophie. Philosophia (Belgrad) 1: 77-176 (1936).

[69] Lycan, L.G. Occam's razor. Metaphilosophy 6(3-4): 223-237 (1975).

[70] Thorburn, W. M. The Myth of Occam's Razor. Mind (New Series) 27(107): 345-353 (1918).

[71] Domingos, P. The Role of Occam's Razor in Knowledge Discovery. Data Mining and Knowledge Discovery 3: 409-425 (Amsterdam: Kluwer, 1999).

[72] Pitt, J. C. (ed.) Change and Progress in Modern Science. Dordrecht, Boston: D. Reidel (1985).

[73] Harisson, P. Experimental religion and experimental science in early modern England. Intellectual History Review 21(4) 2011: 413-433

[74] Russell, B. The philosophy of logical atomism and other essays, 1914-19. London - Boston: George Allen \& Unwin (1986).

[75] Russell, B. (1902) "Letter to Frege," in: J. van Heijenoort (ed.), From Frege to Gödel, Cambridge, Mass.: Harvard University Press, 124-125 (1967). 
[76] Mach, E. Sinnliche Elemente und naturwissenschaftliche Begriffe. Pfliiger's Archiv fur die gesamte Physiologie des Menschen und der Tiere 136: 263-274 (Bohn: Martin Nager, 1910).

[77] Mach, E. Erkenntnis und Irrtum. Skizzen zur Psychologie der Forschung. Leipzig: J. A. Barth (1905).

[78] Mach, E. On the Part Played by Accident in Invention and Discovery (Inaugural lecture delivered on assuming the Professorship of the History and Theory of Inductive Science in the University of Vienna, October 20, 1895). Monist 6(2): 161-175 (1896).

[79] Banks, E. C. Ernst Mach's World Elements. A Study in Natural Philosophy. Dordrech: Kluwer (2003).

[80] Cekic, M. Mach's Phenomenalism as a Link Between Physics and Psychology. In: Blackmore, J. (ed.) Ernst Mach - A Deeper Look. Documents and new perspectives (Boston Studies in Philosophy of Science 143). Amsterdam: Kluwer, 201-204 (1992).

[81] Hamilton, A. Ernst Mach and the Elimination of Subjectivity. Ratio (New Series) 3(2): 117-135 (1990).

[82] Mach, E. Die Prinzipien der physikalischen Optik. Historisch und erkenntnispsychologisch entwickelt. Leipzig: J. A. Barth (1921).

[83] Blackmore, J. Ernst Mach Leaves 'The Church of Physics'. The British Journal for the Philosophy of Science 40(4): 519-540 (1989).

[84] Ленин, В. И. Матеріализмъ и эмпиріокритицизмъ. критическіе заметки объ одной реакиіонной философіи. Москва: Звено (1909).

[85] Honderich, T. (ed.) Oxford companion to philosophy. Oxford: University Press (2005).

[86] Einstein, A. Ernst Mach. Physikalische Zeitschrift 17(01.04.1916): 101-104 (1916).

[87] Holton, G. J. Mach, Einstein, and the Search for Reality. In: Cohen, R. S., Seeger, R. J. (eds.) Ernst Mach: Physicist and Philosopher (Boston Studies in the Philosophy of Sciences, 6). Dordrecht: D. Reidel, 165-199 (1970).

[88] Spiegelberg, H. The Context of the Phenomenological Movement (Phenomenologica 80). The Hague: Martinus Nijhoff (1981).

[89] Spiegelberg, H. The Phenomenological Movement. Dordrecht: Kluwer (1999).

[90] Husserl, E. Über das Prinzip der Vergleichung in der Physik. Archiv für systematische Philosophie. 3: 241-244 (1897).

[91] Mach, E. Über die Vergleichung als wissenschaftliches Prinzip. Verhandlungen der Gesellschaft Deutscher Naturforscher und Ärzte. Theil 1. Leipzig., 44-56 (1894).

[92] Husserl, E., Jerusalem, W. "Husserl vs. Jerusalem," in: Blackmore, J. T., Itagaki, R, Tanaka, S. (eds.) Ernst Mach's Vienna, 1895-1930, or, Phenomenalism as philosophy of science. Dordrecht; Boston: Kluwer (2001), 211-235.

[93] Fissette, D. Phenomenology and Phenomenalism: Ernst Mach and the Genesis of Husserl's phenomenology. Axiomathes 22(1): 53-74 (2012).

[94] Fisette, D. Fenomenologia e fenomenismo em Husserl e Mach. Scientice zudia (São Paulo) 7(4): 535-576 (2009).

[95] Moran, D. Husserl's transcendental philosophy and the critique of naturalism. Continental Philosophy Review 41(4): 401-425 (2008).

[96] Ratliff, F. On Mach's Contributions to the Analysis of Sensations. In: Cohen, R. S., Seeger, R. J. (eds.) Ernst Mach: Physicist and Philosopher (Boston Studies in the Philosophy of Sciences 6). Dordrecht: D. Reidel, 23-41 (1970).

[97] Cohen R. S. Ernst Mach: Physics, Perception and the Philosophy of Science. In: Cohen, R. S., Seeger, R. J. (eds.) Ernst Mach: Physicist and Philosopher (Boston Studies in the Philosophy of Sciences 6). Dordrecht: D. Reidel, 126-164 (1970).

[98] Berg, A. Phenomenalism, phenomenology, and the question of time: a comparative study of the theories of Mach, Husserl, and Boltzmann. Lanham, MD: Lexington Books (2016).

[99] Orilia, F. Singular reference: a descriptivist perspective (Philosophical studies series, 113). Dordrecht: Springer (2010).
[100] Kush, M. Language as calculus vs. language as universal medium: a study in Husserl, Heidegger, and Gadamer (Synthese library, 207). Dordrecht; Boston: Kluwer (1989).

[101] Guzzardi, L. Energy, Metaphysics, and Space: Ernst Mach's Interpretation of Energy Conservation as the Principle of Causality. Science \& Education 23(6): 1269-1291 (2014).

[102] Regt, H. de. "Erwin Schrödinger," in: Blackmore, J. T., Itagaki, R, Tanaka, S. (eds.) Ernst Mach's Vienna, 1895-1930, or, Phenomenalism as philosophy of science. Dordrecht; Boston: Kluwer (2001), 97-103.

[103] "Problems of philosophy." Problem \#19: Phenomenalism vs. phenomenology. Synthese 118(3): 501 (1999).

[104] Banks., E. C. The Philosophical Roots of Ernst Mach's Economy of Thought. Synthese 139(1): 23-53 (2004).

[105] Alten, M. van. Brouwer Meets Husserl. On the phenomenology of choice sequences (Synthese Library 335). Dordrecht: Springer (2007).

[106] Niekus, J. The method of the creative subject. Indagationes Mathematicae (Proceedings) 90(4): 431-443 (1987).

[107] Chaitin, G. J. Randomness in arithmetic and the decline and fall of reductionism in pure mathematics. Chaos, Solitons \& Fractals 5(2): 143-159 (1995).

[108] Agazzi, E. The Problem of Reductionism in Science. Reductionism as Negation of the Scientific Spirit. In: Agazzi, E. (ed.) The Problem of Reductionism in Science: Colloquium of the Swiss Society of Logic and Philosophy of Science, Zürich, May 18-19, 1990. Dordrecht: Springer, 1-29(1991).

[109] Hintikka, J. What is the axiomatic method? Synthese 183(1): 69-85 (2011).

[110] Leng, M. Mathematics and reality. Oxford: University Press, (2010).

[111] Weber, H. Leopold Kronecker. Mathematische Annalen. 43(1): 1-25 (1893).

[112] Einstein, A. Über die spezielle und die allgemeine Relativitätstheorie: (gemeinverständlich) (Sammlung Vieweg, 38). Braunschweig: Friedr. Vieweg \& Sohn, (1917).

[113] Feyerabend, P. K. Mach's theory of research and its relation to Einstein. Studies in History and Philosophy of Science (Part A) 15(1): 1-22 (1984) 
[69] Sörensen, D. Theory formation and the study of literature. Amsterdam: Rodopi (1987).

[70] Zermelo, E. Untersuchungen über die Grundlagen der Mengenlehre I. Mathematische Annalen 65(2): 261-281 (1908).

[71] Popper, K. Logik der forschung: zur erkenntnistheorie der modernen naturwissenschaft. Wien: Springer (1935).

[72] Weber, M. Die 'Objektivität' sozialwissenschaftlicher und sozialpolitischer Erkenntnis. In: Archiv für Sozialwissenschaft und Sozialpolitik 19(1): 22-87 (1904).

[73] Winfired, N. Charles S. Peirce's Theory of Information: A Theory of the Growth of Symbols and of Knowledge. Cybernetics \& Human Knowing 19(1-2) 137-161 (2012)

[74] Peirce, C.S. The Regenerated Logic. The Monist 8(1): 19-40 (1896).

[75] Peano, I. Arithmetices principia: nova method exposita. RomaeFlorentiae: Fratres Bocca (1889).

[76] Lambalgen, M. van. Independence, Randomness and the Axiom of Choice. The Journal of Symbolic Logic 57(4) (1992).

[77] Kingman, J. F. C. Random Discrete Distributions. Journal of the Royal Statistical Society Series B (Methodological), 37(1): 1-22 (1975).

[78] Rozenfeld, B. A. A History of Non-Euclidean Geometry: Evolution of the concept of a geometric space. Berlin, etc.: Springer (1988).

[79] Palermo, J. Apodictic Truth: Husserl's Eidetic Reduction versus Induction. Notre Dame Journal of Formal Logic 19(1): 69-80 (1978).

[80] Gentzen, G. Die Widerspruchsfreiheit der reinen Zahlentheorie. Mathematische Annalen 112(1): 493-565 (1936).

[81] Gentzen, G. Beweisbarkeit und Unbeweisbarkeit von Anfangsfällen der transfiniten Induktion in der reinen Zahlentheorie. Mathematische Annalen 119(1): 140-161 (1943).

[82] Gödel, K. Über formal unentscheidbare Sätze der Principia mathematica und verwandter Systeme I. Monatshefte der Mathematik und Physik, 38(1): 173-198 (1931).

[83] Gentzen, G. Die gegenwärtige Lage in der mathematishen Grundlagenforshung. Forshungen zur Logic und zur Grundlegung der exacten Wissenshaften, Neue Folge 4, 5-18 (Leipzig: Hirzel, 1938).

[84] Hilbert, D. Die Grundlagen der Mathematik. Abhandlungen aus dem Seminar der Hamburgischen Universität, 6: 65-85 (1928).

[85] Einstein, A., Podolsky, B. Rosen, N. Can Quantum-Mechanical Description of Physical Reality Be Considered Complete? Physical Review 47(10): 777-780 (1935).

[86] Penchev, V. Philosphy of quantum information. Volume 1: Gödel and Einstein. Sofia: IPhR - BAN (2009, in Bulgarian).

[87] Penchev, V. Quantum Information as the Information of Infinite Series. PhilSci-Archive: An Archive for Preprints in Philosophy of Science: http://philsci-archive.pitt.edu/11266/1/AISB08wMünchen.pdf

[88] Mindel, A. Quantum mind: the edge between physics and psychology. Portland, OR: Lao Tse Press (2000).

[89] Penrose, R. Shadows of the mind: a search for the missing science of consciousness. Oxford; New York: Oxford University Press (1994).

[90] Casari, E. On Husserl's Theory of Wholes and Parts. History and Philosophy of Logic 21(1): 1-43 (2000).

[91] Penchev, V. The Insolubility of the So-called Gödel's First Incompleteness Theorem. Gödelian and Hilbertian Mathematics Philosophical Alternatives 19(5): 104-119 (2010, in Bulgraian) 